\title{
Excimères ioniques triatomiques d'alcalin gaz rare
}

\author{
Ph. Delaporte, M. Voitik, Ch. Tarras, M. Sentis, O. Uteza, B. Fontaine et B. Forestier \\ Institut de Recherche sur les Phénomènes Hors Equilibre, UMR 138 CNRS, \\ Aix-Marseille I et II, I63 avenue de Luminy, 13288 Marseille cedex 9, France
}

\begin{abstract}
Résumé : Les excimères ioniques triatomiques ont été proposés et observés pour la première fois. L'excitation par faisceau d'électrons de mélanges alcalin (A) gaz rare $(\mathrm{Rg})$ a permis d'observer les émissions de fluorescence dans l'ultraviolet du vide de ces excimères $\operatorname{Rg}_{2}{ }^{+} \mathrm{A}$. Une première approche cinétique a permis de déterminer deux voies de production de ces molécules.
\end{abstract}

\section{LES EXCIMERES IONIQUES}

Depuis que N. Basov [1] et R. Sauerbrey [2] ont proposé d'utiliser des excimères ioniques pour réaliser des lasers dans le domaine spectral de l'ultraviolet du vide (VUV), ces molécules ont fait l'objet de nombreuses recherches expérimentales et théoriques [3]-[5]. En particulier, l'excitation de mélanges alcalin $(\mathrm{A})$ - gaz rare $(\mathrm{Rg})$ a permis de mettre en évidence les émissions de fluorescence VUV liées aux excimères ioniques $\operatorname{Rg}^{+} \mathrm{A}$ de $66,5 \mathrm{~nm}\left(\mathrm{He}^{+} \mathrm{Li}\right)$ à $189,9 \mathrm{~nm}\left(\mathrm{Xe}^{+} \mathrm{Li}\right)$. Des études cinétiques ont permis d'expliquer les principaux mécanismes intervenant lors de la production et la destruction de la molécule $\operatorname{Rg}^{+} \mathrm{A}$ [6]-[8].

Dans la continuité de ces études, nous avons proposé et mis en évidence une nouvelle classe d'excimères ioniques: les molécules triatomiques d'alcalin gaz rare $\operatorname{Rg}_{2}{ }^{+} \mathrm{A}$. Les excimères diatomiques sont formés à partir de l'ion $\mathrm{Rg}^{+}$via la réaction à trois corps

$$
\mathrm{Rg}^{+}+\mathrm{A}+\mathrm{M} \rightarrow \mathrm{Rg}^{+} \mathrm{A}+\mathrm{M}
$$

et nous avons supposé que l'ion moléculaire $\operatorname{Rg}_{2}{ }^{+}$, qui est présent dans la cinétique des excimères diatomiques [8], pourrait jouer ce rôle de précurseur dans la formation de l'excimère ionique triatomique $\operatorname{Rg}_{2}{ }^{+} \mathrm{A}: \mathrm{Rg}_{2}{ }^{+}+\mathrm{A}+\mathrm{M} \rightarrow \mathrm{Rg}_{2}{ }^{+} \mathrm{A}+\mathrm{M}$

Le dispositif d'excitation est un faisceau d'électrons permettant la création d'une densité de courant de $1 \mathrm{~A} / \mathrm{cm}^{2}$ durant $800 \mathrm{~ns}$ au niveau de l'axe optique. Le volume excité transversalement par ces électrons est de $1,5 \times 1,5 \times 15 \mathrm{~cm}^{3}$. La densité d'alcalin dans la cellule est contrôlée par la température du mélange, qui peut être chauffé uniformément dans le volume excité jusqu'à $500^{\circ} \mathrm{C}$. Après un hublot en $\mathrm{MgF}_{2}$, un dispositif optique constitué d'un monochromateur, d'un analyseur spectral multicanaux (OSMA) et d'un photomultiplicateur VUV permet d'enregistrer les spectres des émissions de fluorescence et de suivre leurs évolutions temporelles à une longueur d'onde donnée.

\section{ETUDE SPECTROSCOPIQUE ET CINETIQUE}

Afin d'étudier les émissions de fluorescence de molécules ioniques triatomiques d'alcalin gaz rare, nous avons excité par faisceau d'électrons des mélanges $\mathrm{Ar} / \mathrm{Rg} / \mathrm{A}(\mathrm{Rg}=\mathrm{Kr}$, Xe et 
$\mathrm{A}=\mathrm{Cs}, \mathrm{Rb}$ ) à des pressions de gaz rare plus élevées que lors des études sur les ions $\mathrm{Rg}^{+} \mathrm{A}$. Les spectres de $\mathrm{Kr}_{2}{ }^{+} \mathrm{Cs}$ à $159 \mathrm{~nm}$ (figure 1), de $\mathrm{Kr}_{2}{ }^{+} \mathrm{Rb}$ à $160 \mathrm{~nm}$, de $\mathrm{Xe}_{2}{ }^{+} \mathrm{Cs}$ à $190 \mathrm{~nm}$ et de $\mathrm{Ar}_{2}{ }^{+} \mathrm{Cs}$ à $135 \mathrm{~nm}$ ont été observés. Sur la figure 1 , on observe une émission de largeur spectrale relativement étroite $(1,5 \mathrm{~nm})$ centrée à $132 \mathrm{~nm}$ qui correspond à la fluorescence de l'excimère ionique diatomique $\mathrm{Kr}^{+} \mathrm{Cs}$. L'émission du second continuum du krypton $\mathrm{Kr}_{2}{ }^{*}$ apparait à $145 \mathrm{~nm}$ lorsque la quantité de gaz rare est importante et que la pression de vapeur d'alcalin n'est pas suffisante pour la bloquer. Enfin, aux longueurs d'onde encore plus élevées, l'émission de l'excimère ionique triatomique $\mathrm{Kr}_{2}{ }^{+} \mathrm{Cs}$ est identifiable. Cette dernière émission présentent une largeur à mi-hauteur d'environ $20 \mathrm{~nm}$. Les premières analyses spectroscopiques nous laissent supposer que le niveau fondamental de ces transitions est répulsif et nous ont permis d'estimer l'énergie de liaison de $\mathrm{Kr}_{2}{ }^{+} \mathrm{Cs}$ a environ $0,9 \mathrm{eV}$.

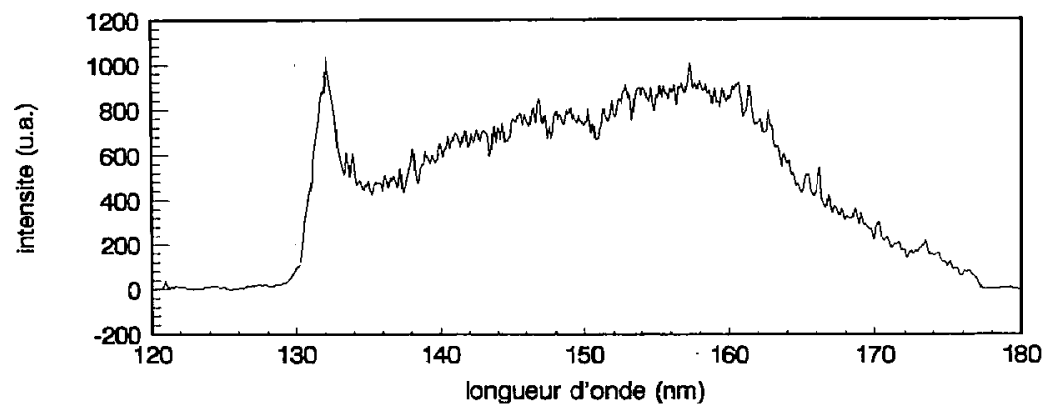

Figure 1 : Spectre enregistré entre $120 \mathrm{~nm}$ et $180 \mathrm{~nm}$ lors de l'excitation par faisceau d'électrons d'un mélange $\mathrm{Ar} / \mathrm{Kr} / \mathrm{Cs}(450 \mathrm{mb} / 240 \mathrm{mb} / 4,1 \mathrm{mb})$

Nous avons constaté que de fortes pressions de gaz rare $\left(\mathrm{P}_{\mathrm{Rg}}>100 \mathrm{mbar}\right)$ favorise la production de $\mathrm{Rg}_{2}{ }^{+} \mathrm{A}$ et de $\mathrm{Rg}_{2}{ }^{*}$ par rapport à $\mathrm{Rg}^{+} \mathrm{A}$ et que l'augmentation de la densité d'alcalin dans le mélange conduit au blocage de l'émission de $\mathrm{Rg}_{2}{ }^{*}$ et à un meilleur rendement de production de $\mathrm{Rg}_{2}{ }^{+} A$ et $\mathrm{Rg}^{+} \mathrm{A}$. A partir de ces évolutions nous avons pu établir un premier schéma cinétique sur la formation et la désexcitation de l'excimère $\mathrm{Rg}_{2}{ }^{+} \mathrm{A}$. Notamment, deux voies de production de ces nouveaux ions triatomiques sont proposées :

$$
\begin{aligned}
& \mathrm{Rg}^{+} \mathrm{A}+2 \mathrm{Rg} \rightarrow \mathrm{Rg}_{2}{ }^{+} \mathrm{A}+\mathrm{Rg} \\
& \mathrm{Rg}_{2}{ }^{+}+\mathrm{A}+\mathrm{Rg} \rightarrow \mathrm{Rg}_{2}{ }^{+} \mathrm{A}+\mathrm{Rg}
\end{aligned}
$$

Cette première approche cinétique, effectuée dans le cas de l'ion $\mathrm{Kr}_{2}{ }^{+} \mathrm{Cs}$, nous a permis d'estimer la durée de vie d'émission spontanée à $5 \mathrm{~ns}$, et les constantes cinétiques des réactions de formation à $5.10^{-30} \mathrm{~cm}^{6} / \mathrm{s}$ pour $\mathrm{k}_{1}$ et $10^{-30} \mathrm{~cm}^{6} / \mathrm{s}$ pour $\mathrm{k}_{2}$.

\section{Références}

[1] N. G. Basov, M. G. Voitik, V. S. Zuev, and V. P. Kutakhov, Sov. J. Quant, Electr 15, 1455 (1985)

[2] R. Sauerbrey and H. Langhoff, IEEE J. Quant. Electron. vol. QE-21, pp. 179-181, 1985

[3] Da Xing, Ken-ichi Ueda, and Hiroshi Takuma, Appl. Phys Lett. 59, 1028 (1991)

[4] H. M. J. Bastiaens et al., Appl. Phys. Lett. 60, 2834 (1992)

[5] P. Millar et al., Appl. Phys. Lett. 55, 2176 (1989)

[6] M. Schumann, H. Langhoff, J. Chem. Phys. 101, 4769 (1994)

[7] H. Tischler, Ph. Delaporte, B. Fontaine, M. Sentis, IEEE. Journal of selected topics in Quantum Electronics, Vol. 1, N³, pp 877 - 885 (1995)

[8] H. Tischler, Ph. Delaporte, and M. Sentis, IEEE. Journal of selected topics in Quantum Electronics, Vol. 1, N³, pp $886-890,1995$ 